\title{
A common variant of the NOTCH4 gene modulates functional connectivity of the occipital cortex and its relationship with schizotypal traits
}

Xiaohui Xie ${ }^{1+}$, Meidan $\mathrm{Zu}^{1 \dagger}$, Long Zhang ${ }^{1+}$, Tongjian Bai ${ }^{1}$, Ling Wei ${ }^{1,2,3}$, Wanling Huang ${ }^{1}$, Gong-Jun Ji2,3,4, Bensheng Qiu', Panpan Hu ${ }^{1,2,3}$, Yanghua Tian ${ }^{1,2,3}$ and Kai Wang ${ }^{1,2,3,4^{*}}$

\begin{abstract}
Background: Schizotypal traits are considered as inheritable traits and the endophenotype for schizophrenia. A common variant in the NOTCH4 gene, rs204993, has been linked with schizophrenia, but the neural underpinnings are largely unknown.

Methods: In present study, we compared the differences of brain functions between different genotypes of rs204993 and its relationship with schizotypal traits among 402 Chinese Han healthy volunteers. The brain function was evaluated with functional connectivity strength (FCS) using the resting-state functional magnetic resonance image(rs-fMRI). The schizotypal traits were measured by the schizotypal personality questionnaire (SPQ).

Results: Our results showed that carriers with the AA genotype showed reduced FCS in the left occipital cortex when compared with carriers with the AG and GG genotypes, and the carriers with the AG genotype showed reduced FCS in the left occipital cortex when compared with carriers with the GG genotype. The FCS values in the left occipital lobe were negatively associated with the SPQ scores and its subscale scores within the carriers with the GG genotype, but not within the carriers with AA or AG genotype.
\end{abstract}

Conclusion: Our results suggested that the common variant in the NOTCH4 gene, rs204993, modulates the function of the occipital cortex, which may contribute to schizotypal traits. These findings provide insight for genetic effects on schizotypal traits and its potential neural substrate.

Keywords: NOTCH4, rs204993, Functional connectivity strength, Occipital cortex, Schizotypal traits

\footnotetext{
*Correspondence: wangkai1964@126.com

${ }^{+}$Xiaohui Xie, Meidan Zu and Long Zhang contributed equally to this work.

'Department of Neurology, The First Affiliated Hospital of Anhui Medical University, Hefei 230022, China

${ }^{2}$ Collaborative Innovation Center for Neuropsychiatric Disorders and Mental Health, Anhui Medical University, Hefei 230022, China

Full list of author information is available at the end of the article
}

(c) The Author(s). 2020 Open Access This article is licensed under a Creative Commons Attribution 4.0 International License, which permits use, sharing, adaptation, distribution and reproduction in any medium or format, as long as you give appropriate credit to the original author(s) and the source, provide a link to the Creative Commons licence, and indicate if changes were made. The images or other third party material in this article are included in the article's Creative Commons licence, unless indicated otherwise in a credit line to the material. If material is not included in the article's Creative Commons licence and your intended use is not permitted by statutory regulation or exceeds the permitted use, you will need to obtain permission directly from the copyright holder. To view a copy of this licence, visit http://creativecommons.org/licenses/by/4.0/ The Creative Commons Public Domain Dedication waiver (http://creativecommons.org/publicdomain/zero/1.0/) applies to the data made available in this article, unless otherwise stated in a credit line to the data. 


\section{Background}

Schizophrenia is a chronically severe, mental disorder, affecting approximately $1 \%$ of the worldwide population [1]. In addition, some individuals present preclinical symptoms of schizophrenia called schizotypal traits [2]. Schizotypal traits refer to a set of traits, continually distributed in the general population, that resemble some of the symptoms of schizophrenia. Schizotypal traits provide important insights into understanding the origins of schizophrenia [3]. Raine et al. concluded that structural and functional alterations in the brain induced by genetic and early environmental influences contribute to the development of schizotypy [4]. Indeed, schizotypal traits are more frequent in first-degree relatives of patients with schizophrenia, suggesting a genetic link between schizotypal traits and schizophrenia $[5,6]$. The schizophreniarisk gene also contributes to the presence of schizotypal traits $[7,8]$. Hence, the schizophrenia-risk gene may have potential modulatory effects on schizotypal traits.

Although previous studies have found that some genes indicate susceptibility for schizophrenia [9, 10], only a small percentage of the genes have been consistently studied among different racial populations. The NOTCH4 (neurogenic locus notch homolog protein4) gene, located in the major histocompatibility complex (MHC) region of 6 p21.3 in humans, is highly associated with schizophrenia among different racial populations [11-13]. Wei and Hemmings found that the NOTCH4 locus was involved in susceptibility to schizophrenia in a British population [13]. The relationship between schizophrenia and the common variants of NOTCH4 gene was also found among different racial populations (the rs2071287 and rs3131296 in European and Japanese population) $[14,15]$. However, these SNPs were not confirmed among Chinese populations. Recently, a study among 218 Taiwanese families reported another SNP, rs204993 with a significant relationship with schizophrenia, found by testing the association of the entire genomic region of NOTCH4 [16]. Specially, the AA genotype of rs204993 was associated with a higher risk for schizophrenia in the Chinese Han population [17]. These findings suggested the common variant of the NOTCH4 gene, SNP rs204993 may be a potential genetic basis for schizophrenia in the Chinese population.

Recent evidences suggested the intermediary role of brain function for the genetic effect on schizophrenia or schizotypal traits. However, the effect of the common variant of the NOTCH4 gene, SNP rs204993, on brain function and its relationship with schizophrenia trait has not been investigated. Imaging genetics may provide a neural basis to explain how the gene affects the occurrence and development of diseases via connecting the link between gene and brain diseases [18]. Resting-state functional magnetic resonance imaging (rsfMRI), as a task-free and noninvasive measurement tool, has been used in several mental disorders [19]. Functional connectivity strength (FCS), which identifies functional hubs of human brain networks based on the graph theory method [20], is a developed method to test the connectivity of each voxel with all other voxels in the brain [21]. The FCS analysis has been used to characterize the neural mechanism in various psychiatric disorders [22], including schizophrenia and schizotypal traits [23, 24]. For example, decreased FCS in the bilateral occipital cortex and the right sensorimotor cortex as well as increased FCS in the bilateral temporal cortex, hippocampus, and the left prefrontal cortex were observed in schizophrenia [21]. Moreover, schizotypal traits presented abnormal connectivity in the default mode network compared with healthy controls [24].

In present study, we aimed to investigate the genetic effect of the schizophrenia-related gene (NOTCH4 rs204993 genotype) on brain function and its relationship with schizotypal traits among healthy volunteers. Based on the above findings, we propose that the schizophrenia-risk rs204993 genotype (AA genotype) may be linked with attenuated function in schizophrenia-related brain regions, which contributes to schizotypal traits.

\section{Methods}

\section{Participants}

We recruited 641eligible healthy Chinese Han participants from the Anhui Medical University in Hefei, Anhui province. The inclusion criteria included: 1) undergraduate students; 2) the participant and whose parents were the Chinese Han populations; 3) age 18-25 years old; 4) Voluntary to be taken blood sample and MRI scanning; 5) no contraindication for MRI scanning. Exclusion criteria included: Psychiatric disorders, neurological diseases, alcohol or drug abuse, brain injury, visible brain structure abnormities, or excessive motion during MRI scanning $(>2.0 \mathrm{~mm}$, $\left.2.0^{\circ}\right)$. Participants with a first-degree relative with a neurological or psychiatric disorder were also excluded. Of these participants, a total of 239 participants were excluded because of NOTCH4 genotyping failure (12 participants) or poor MRI data (198 participants with excessive head motion and 29 participants with artifact in their structural images). Finally, a total of 402 participants were included in the data analysis of the current study. The study was approved by the Anhui Medical University Ethics Committee, and all participants signed informed consent forms after being given a thorough description of the study.

\section{SNP genotyping}

Blood samples were collected to obtain DNA using standard procedures. The genotyping of all participants was determined in comparison with control DNA confirmed by sequencing in the SNP pattern. We used the Light Scanner Primer Design software program (Idaho 
Technology, UT, USA) to design the primer. The PCR primers were designed to amplify the desired locus, within which the target SNP (rs204993 in NOTCH4 gene) is located: 5' -TTCGGGACTTCTGTTCAGCC-3' (forward), 5'-TCCTGGAAGCACTCGTTGAC-3' (reverse). Then, the amplicon library was used for highthroughput sequencing by Illumina HiSeq X Ten (Illumina, San Diego, CA, USA). Finally, the sequence reads were mapped to the human reference genome GRCH37 and the genotype of NOTCH4 rs204993 was divided into the AA, GA, and GG genotypes.

\section{Schizotypal trait evaluations}

The schizotypal traits were evaluated using the schizotypal personality questionnaire (SPQ) [25]. The SPQ includes a 74-item with a "yes/no" response to assess schizotypal traits in the general population. The questionnaire subscale scores reflect different schizotypal dimensional features, including interpersonal, cognitive perceptual, and disorganized factors [26]. In the present study, we used the Chinese version SPQ, which has good internal consistency (0.95) and reliability value $(0.86)$ for the Chinese population [27].

\section{Image data acquisition}

Structural and functional MRI images of participants were acquired under a 3-T scanner at the University of Science and Technology of China, Anhui Province. Before images acquisition, all participants were asked to keep eyes closed and body still, and not to think of anything particularly. T1-weighted anatomical images with were acquired in sagittal orientation $(\mathrm{TR} / \mathrm{TE}=8.16 / 3.18$ ms; flip angle $=12^{\circ}$; field of view $(\mathrm{FOV})=256 \times 256 \mathrm{~mm}^{2}$; voxel size $=1 \times 1 \times 1 \mathrm{~mm}^{3}$; slice thickness $=1 \mathrm{~mm} ; 188$ slices). Functional MRI (BOLD) images were composed of 217 echo-planar imaging volumes $(\mathrm{TR} / \mathrm{TE}=2400 / 30$ ms; flip angle $=90^{\circ} ; \mathrm{FOV}=192 \times 192 \mathrm{~mm}^{2}$; voxel size $=$ $3 \times 3 \times 3 \mathrm{~mm}^{3}$; slice thickness $=3 \mathrm{~mm}$; matrix size $=64 \times$ 64 ; and 46 continuous slices).

\section{Functional image preprocessing}

The Data Processing Assistant for Resting-State Functional MRI imaging toolkit (DPARSF) was used for the functional image preprocessing, which was based on the following software: the Resting State Functional MR Imaging Toolkit and statistical parametric mapping software package (SPM8) [28, 29]. We discarded the first five volumes of data to ensure stable longitudinal magnetization, and the remaining volumes were corrected for slice timing and head motion. Next, we normalized the structural T1 image to the Montreal Neurological Institute space based on the T1 image unified segmentation with a 12 parameter nonlinear transformation. Finally, the data were nuisance regressed with 24 Friston motion parameters, white matter high signals, cerebrospinal fluid signals, and global signals as regressors, and filtered with a temporal bandpass of $0.01-0.1 \mathrm{~Hz}$.

\section{Whole brain FCS analysis}

A voxel-wise, whole-brain functional connectivity analysis was performed on the preprocessed rsfMRI data as in our previous study [30]. First, Pearson's correlations between the residual time series of all pairs of brain voxels were computed and a whole brain connectivity matrix for each participant was constructed. The individual correlation matrices were then transformed to a z-score matrix using a Fisher r-to-z transformation. For a given voxel, FCS was computed as the sum of the $\mathrm{z}$-values between the given voxel and all other voxels. We restricted our analyses to correlations above a threshold of $r=0.1$ to eliminate weak correlations possibly arising from noises. These FCS maps were smoothed with a $4 \mathrm{~mm}$ full-width at a halfmaximum (FWHM) Gaussian kernel [20, 31].

\section{Statistical analysis}

The $\chi^{2}$ test was used to compare the sex differences, and one-way analysis of variance (ANOVA) was used to check for differences in age, educational years and SPQ among the three genotype groups (AA, AG and GG). Although there is study has suggested a recessive effect (AA) of rs204993, there is no consensus about analytically grouping the genotypes. Hence, we performed with an unbiased approach that of three possible effects were all considered: additive (GG versus GA versus AA genotypes), dominant (A allele carriers versus $\mathrm{GG}$ ); recessive (AA versus $\mathrm{G}$ allele carriers). For additive effect, one-way ANOVA was performed to determine the changed regions of FCS for healthy participants among the three genotype groups. For dominant or recessive effect, two sample t-tests were performed (A allele carriers versus GG, AA versus G allele carriers). There tests were constrained in a gray matter mask, and multiple comparison corrections were based on a Gaussian random field theory (voxel-level $p<0.001$; cluster-level $p<0.05$ ). The FCS value of the changed regions was extracted and compared with one-way ANOVA among the three genotype groups, and post hoc analysis was conducted using the LSD method. Spearman's correlation analyses were performed to assess the correlation between the values of FCS and the behavior scores (total SPQ scores and its sub-domains) for each NOTCH4 genotype group (the significance level: $p<0.05 / 5$, two-tail).

\section{Results}

Demographic information and the genetic effect on behavior performance

Table 1 shows the demographic, genetic, and behavioral data for participants included in the final analysis. The 
Table 1 Demographic and behavioral-neuroimgaing information for participants grouped by the rs204993 genotype (mean \pm SD)

\begin{tabular}{llllll}
\hline & AA & AG & GG & Statistics & $P$ value \\
\hline Number of participants & 142 & 188 & 72 & $/$ & 2.250 \\
Sex (male/female) & $68 / 74$ & $88 / 100$ & $20.942 \pm 1.061$ & $21.097 \pm 1.115$ & 2.490 \\
Age (years) & $20.761 \pm 1.104$ & $26.553 \pm 14.038$ & $31.167 \pm 14.470$ & 2.955 & 0.325 \\
SPQ & $26.930 \pm 13.853$ & $0.612 \pm 0.531$ & $0.755 \pm 0.542$ & 15.354 \\
FCS in occipital cortex & $0.379 \pm 0.461$ & & 0.051 \\
\hline
\end{tabular}

SPQ schizotypal personality questionnaire, FCS functional connectivity strength, SD standard deviation

genotype distribution of NOTCH4 rs204993 in the current study $(\mathrm{AA}=142, \mathrm{AG}=188$, and $\mathrm{GG}=72)$ was consistent with a previous report of variations of this gene in the healthy Chinese Han population [17]. Genotype groups did not significantly deviate from Hardy-Weinberg equilibrium $\left(\chi^{2}=0.505, p=0.477\right)$. There was no significant difference in sex, educational years, and age between the genotype groups. There was also no significant difference of SPQ between the genotype groups.

\section{The genetic effect of rs204993 on FCS}

After controlling for demographic factors, only the additive effect (GG versus GA versus AA genotypes) was survived after corrected using Gaussian random field theory (voxel-level $p<0.001$; cluster-level $p<0.05$ ). The results showed that FCS was significantly abnormal in the left occipital cortex among the NOTCH4 genotypes (Fig. 1). The FCS values of the above cluster were then extracted in the three genotype groups. We found a main effect of the genotype on FCS values $(F=15.354$,

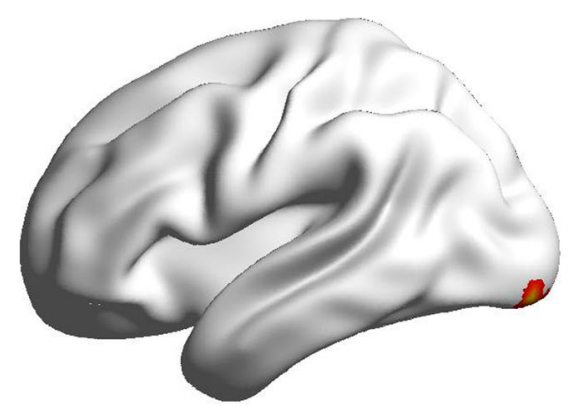

F value
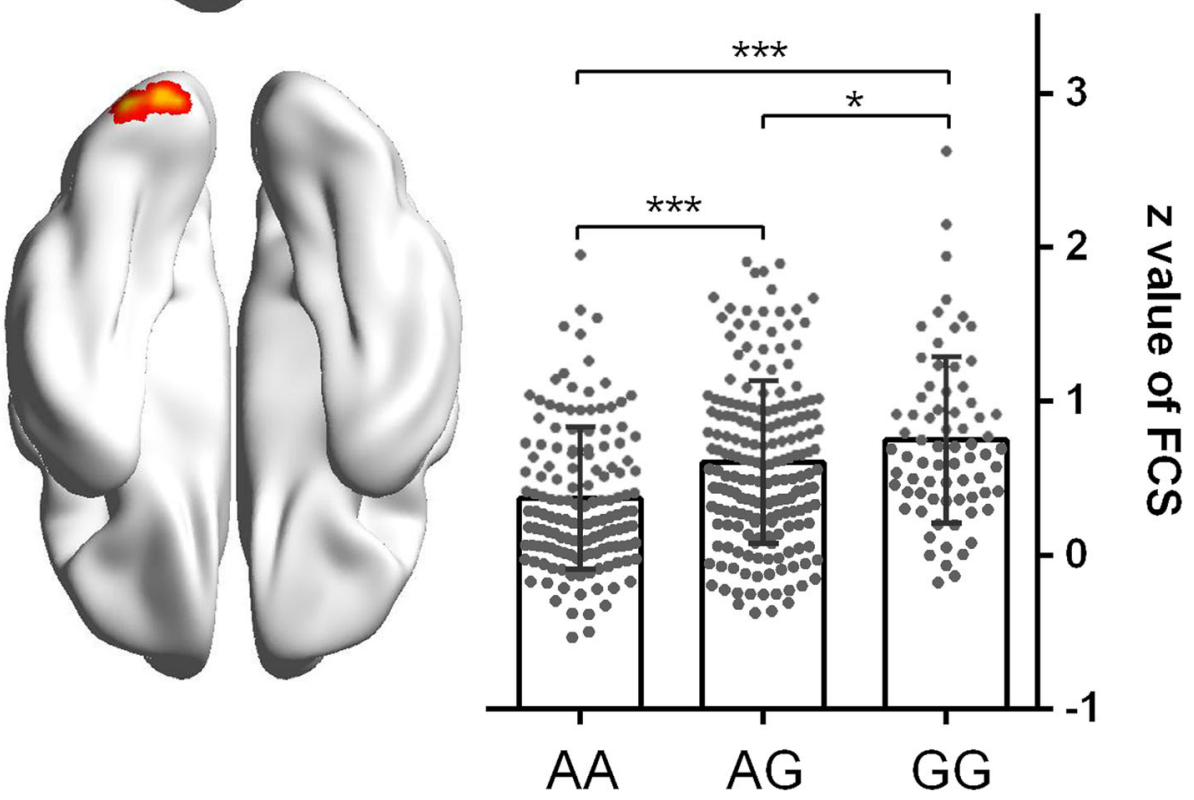

Fig. 1 Modulatory effect of rs204993 on the functional strength (FCS). The X-axis shows the different genotypes, and the Y-axis shows the mean \pm SD of the FCS in the occipital cortex. Group comparisons revealed that the FCS in the occipital cortex was the highest in GG individuals and decreased from GG and AG individuals to AA individuals. All thresholds for comparisons were set as a whole brain Gaussian random field (GRF) correction (voxel $P<0.001$, cluster $P<0.05$ ) 
$p<0.001)$. Post hoc analysis showed AA individuals having reduced values when compared with AG and GG participants ( $p<0.001$ for both) and AG individuals having reduced values when compared with GG partici$\operatorname{pants}(p=0.05) \quad$ (Fig. 1 and Table 1). No cluster was survived after GRF multiple comparison corrections based dominant or recessive statistical model.

\section{Correlation analyses between SPQ and FCS}

The FCS values in the left occipital cortex were negatively associated with the total SPQ scores $(r=-0.391, p=0.001)$, cognitive-perceptual scores $(r=-0.411, p<0.001)$, interpersonal scores $(r=-0.342, p=0.003)$ and disorganized scores ( $r=-0.233, p=0.049$ ) among GG participants (Fig. 2). No significant relationship was found between the values of FCS and the behavior scores (total SPQ scores and its subdomains) for the AA or AG group (Fig. 3).

\section{Discussion}

In the current study, we aimed to investigate the genetic effect of the schizophrenia-related gene (NOTCH4 rs204993 genotype) on brain function and its relationship with schizotypal traits among healthy volunteers. Using a large sample size, we found that functional connectivity in the occipital cortex was the highest in GG individuals and decreased from GG and AG individuals to AA individuals. Notably, the values of FCS in the left occipital cortex were negatively correlated with total SPQ scores and its subset scores (cognitive-perceptual and interpersonal scores) in GG individuals.

Previous studies have reported that NOTCH4 polymorphism of rs204993 is closely related to schizophrenia $[12,13]$. However, as a candidate gene for schizophrenia, we did not find a direct effect of NOTCH4 rs204993 on the schizotypal traits among healthy volunteers. Even so, it was notable that we found the modulatory effect of
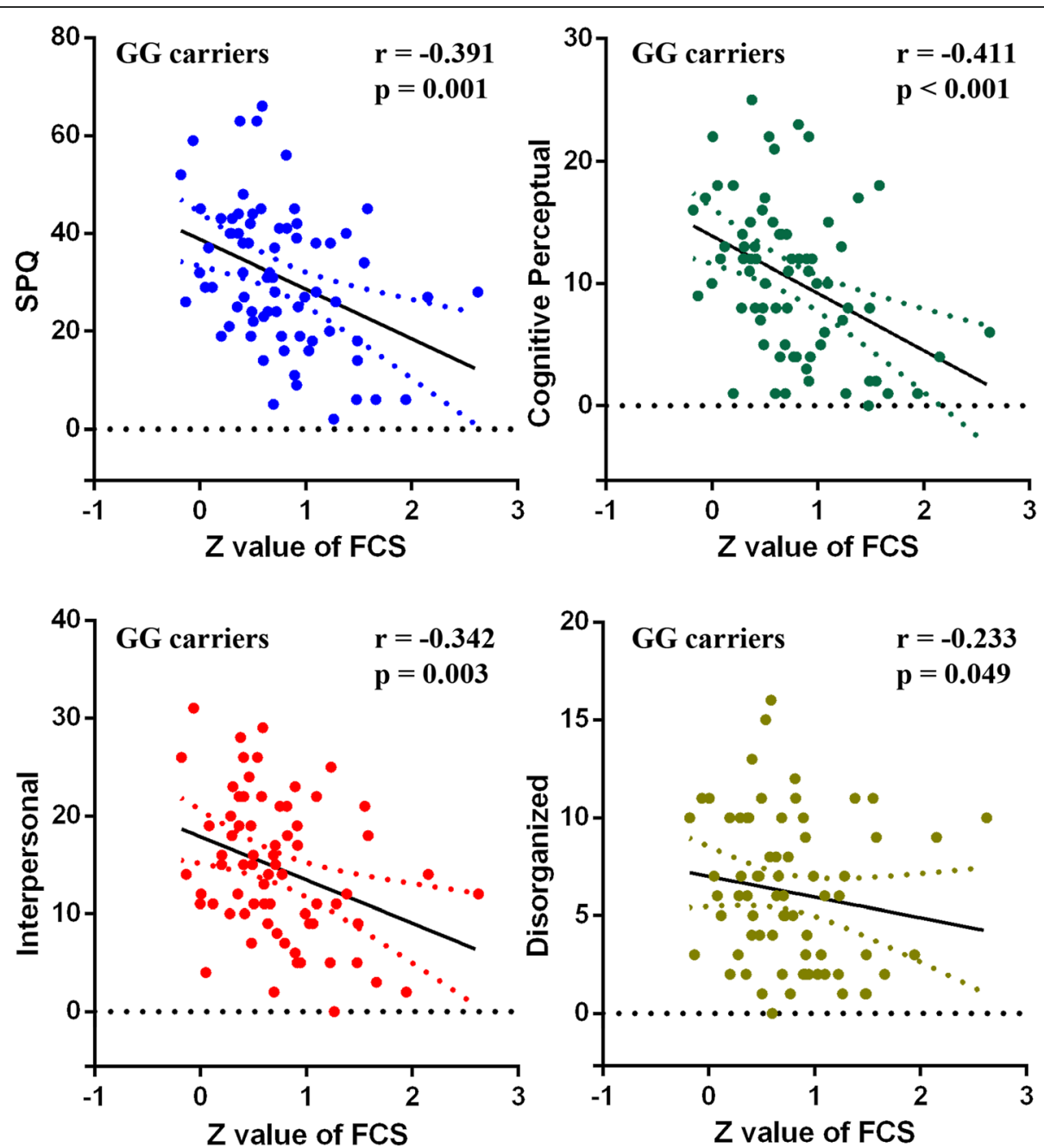

Fig. 2 Relationship between the functional strength (FCS) in the occipital cortex and schizotypal traits among the GG genotype carriers. The FCS in the occipital cortex was negatively correlated with the score of schizotypal personality questionnaire (SPQ) and its subscale scores (cognitive perceptual and interpersonal) among the GG genotype carriers). Note: ${ }^{* * *} p<0.001,{ }^{*} p<0.05$ 

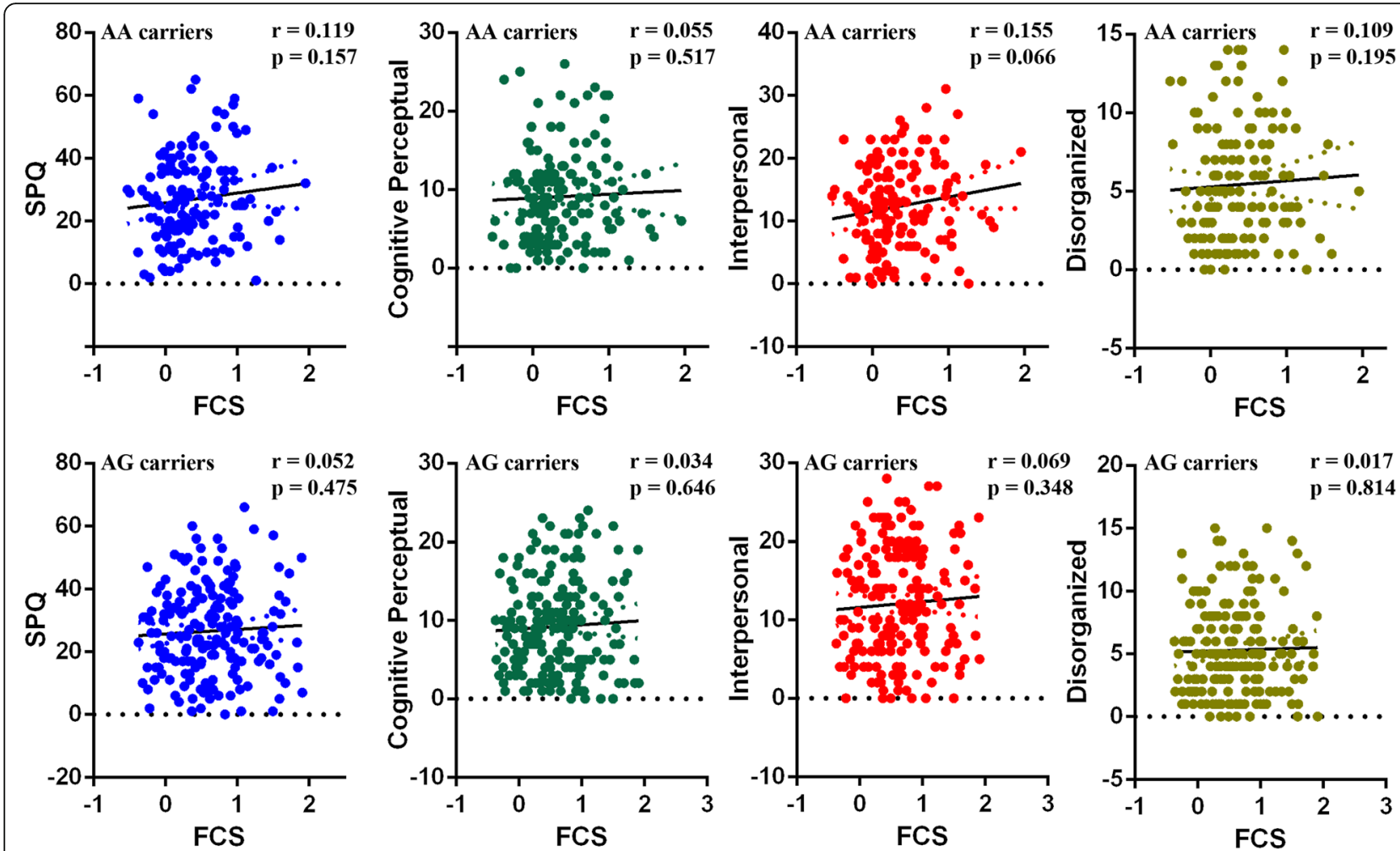

Fig. 3 Relationship between the functional strength (FCS) in the occipital cortex and schizotypal traits among the AA and AG genotype carriers. No significant correlation was found between the FCS in the occipital cortex and the schizotypal personality questionnaire scores or its subscale scores

NOTCH4 rs204993 on brain function (the functional connectivity of occipital cortex), which was associated with schizotypal traits. The impact of the NOTCH4 gene on the central nervous system has been consistently reported [32]. The NOTCH4 gene is one member of the $\mathrm{NOTCH}$ family, which may code for the notch protein, which was identified as a Drosophila neurogenic protein during embryogenesis [33]. Functionally, the NOTCH family mainly controls the cell fate in the process of neurodevelopment, which promotes proliferative signaling $[34,35]$. Therefore, the NOTCH4 gene variants may potentially affect the function or expression level of the Notch4 protein, which in turn influences the neurodevelopment of certain psychiatric disorders, such as schizophrenia and schizotypal traits. In the present study, we found an aberrant functional connectivity in the left occipital cortex among carriers with the NOTCH4 rs204993 schizophrenia-risk genotypes.

The occipital cortex plays an important role in the neural circuits of perceptual processing, such as visual and auditory processing $[36,37]$. Considerable studies have confirmed that schizophrenia patients and their unaffected siblings present with impaired perceptual performance, which is associated with impaired structural and functional connectivity in the occipital cortex [38-40].
Furthermore, the occipital lobe has also been gradually recognized as the neural basis for perceiving personal communication and social interactions [41-43]. Of course, some studies have shown that negative symptoms in patients with schizophrenia are associated with abnormal changes in the occipital cortex [44-46]. For example, abnormal connectivity between the occipital cortex and ventral tegmental area is related to the negative symptoms in schizophrenia [44]. In general, the occipital cortex may be a common neural pathway for the processing of positive and negative symptoms in schizophrenia. Consistent with this possibility, our study showed that the occipitalcortex functional connectivity was related with cognitiveperceptual and interpersonal subscores, which represented the positive and negative symptoms in schizotypal traits. We therefore speculate that the NOTCH4 gene may induce the occurrence and development of diseases by affecting the neurodevelopment of occipital cortex.

It is worth noting that we found that the FCS of the occipital cortex was degressive in sequences of the GG, AG, and AA genotype groups that suggest an additive effect of rs204993. This is not in complete agreement with a recessive effect revealed by the previous results that the AA genotype of rs204993 is associated with a higher risk for schizophrenia in the Chinese Han 
population [17]. The difference between schizophrenia and schizotypal traits may explain this discrepancy. As our results suggested, individuals with the AG genotype also presented lower FCS in the occipital cortex, when compared with the GG genotype carriers, which may indicate the risk of schizotypal traits in the AG genotype carriers, but not the risk of schizophrenia.

It is interesting that the correlation between occipitalcortex function and SPQ is restricted to specific genotype. Detailedly, individual occipital-cortex FCS was inversely correlated with individual SPQ and its subdomains (cognitive-perceptual and interpersonal scores) only for GG carriers who may present lower risk for schizophrenia. These results implicate a modulation of occipital-cortex function with cognitive-perceptual and interpersonal ability that the individuals with higher occipital-cortex FCS present more normal cognitiveperceptual and interpersonal ability, which are featured impairments in schizophrenia. The disappearance of such correlation may suggest an impaired modulation of occipital-cortex function with cognitive-perceptual and interpersonal ability in among relative high-risk individuals (AA and AG carriers). Similarly, this genotypedepended correlation has been reported by previous genetic imaging study [47]. Another interesting finding of the present study was that the significant difference among groups was only located at the left occipital cortex and not the right. We speculate that the lefthemisphere dominant is the potential factor underlying this lateralization. Although we did not collect the handedness data, based on a survey reported about 87.794.8\% right hand domination in Chinese population [48], we believe that most participants in present study are left-hemisphere dominant. The left occipital cortex may prefer to involve in mental and emotional processing, hence, left occipital-cortex connective decline may more contribute to schizophrenia symptoms.

Several potential limitations of our study need to be considered. First, we did not find a direct modulatory effect of the NOTCH4 gene on schizotypal traits (no significant difference between NOTCH4 genotype groups). The gross feature of the schizotypal-trait assessment (SPQ) may partly explain this defect. Second, the absence of long scanning durations reduced the reliability of measuring functional connectivity. Third, our study did not reveal the cognitive mental mechanism of NOTCH4 gene modulating the occipital cortex and its relationship with schizotypal traits. Fourth, previous studies confirmed the relationship between schizophrenia and NOTCH4 polymorphisms, including single nucleotide polymorphisms (SNPs) (e.g., rs520692, rs3131296, rs204993, and rs2071287). But we only explored the relationship between FCS of left occipital cortex and the NOTCH4 rs204993 genotype in populations with schizotypal traits. Further studies are needed to analyze the associations with other SNPs of the NOTCH4 gene. Finally, we did not characterize the modulatory effect of the $\mathrm{NOTCH} 4$ gene on local and long-range functional connectivities, respectively, which may represent distinct biological differences.

\section{Conclusion}

Our results suggested that the common variant in the NOTCH4 gene, rs204993, modulates the function of the occipital cortex, which may contribute to schizotypal traits. These findings provide insight for genetic effects on schizotypal traits and its potential neural substrate.

\section{Abbreviations}

FCS: Functional connectivity strength; SPQ: Schizotypal Personality Questionnaire; rs-fMRl: Resting-state functional magnetic resonance image; NOTCH4: Neurogenic locus notch homolog protein4; MHC: Major histocompatibility complex; SNPs: Single nucleotide polymorphisms; DPARSF: Data Processing Assistant for Resting-State Functional; SPM8: Statistical parametric mapping software package; REST: Resting State Functional MR Imaging Toolkit; FWHM: Full-width at half-maximum

\section{Acknowledgements}

We thank all the participants from Anhui Medical University and operators of the Center for Biomedical Engineering, University of Science and Technology of China for their support of this study.

\section{Authors' contributors}

$X H X, M D Z, L Z$, and KW designed the study. LZ, TJB, WLH, and PPH acquired the behavioral and imaging data. LW and GJJ collected and analyzed the genetic data. TJB, GJJ, BSQ, and YHT analyzed the imaging data. XHX, MDZ, $\mathrm{LZ}$, and KW wrote this article, which all authors reviewed. All authors approved the final version to be published, and we certify that no other individuals not listed as authors have made substantial contributions to the paper.

\section{Funding}

This study was supported by the National Natural Science Foundation of China $(31970979,91432301,31571149,81171273,91232717$ to K.W. 81671354 and 91732303 to Y.T., and 31800909 to L.Z.). The funding agency had no role in the study design, data collection and analysis, or preparation of the manuscript.

\section{Availability of data and materials}

Genetic data in this article are deposited in the NCBI SRA database (accession: SRP268121). Requests to access the MRI data should be addressed to wangkai1964@126.com.

\section{Ethics approval and consent to participate}

All participants signed the informed consent. The study adhered to the tenets of the Declaration of Helsinki and was approved by the Biomedical Ethics Committee of Anhui Medical University.

Consent for publication

Not applicable.

Competing interests

All authors declare no competing interests.

\section{Author details}

${ }^{1}$ Department of Neurology, The First Affiliated Hospital of Anhui Medical University, Hefei 230022, China. ${ }^{2}$ Collaborative Innovation Center for Neuropsychiatric Disorders and Mental Health, Anhui Medical University, Hefei 230022, China. ${ }^{3}$ Anhui Province Key Laboratory of Cognition and Neuropsychiatric Disorders, Hefei 230022, China. ${ }^{4}$ Department of Medical Psychology, Chaohu Clinical Medical College, Anhui Medical University, Hefei, China. ${ }^{5}$ Center for Biomedical Engineering, University of Science and Technology of China, Hefei, Anhui, China. 
Received: 9 January 2020 Accepted: 29 June 2020

\section{Published online: 09 July 2020}

\section{References}

1. Andreasen NC. Symptoms, signs, and diagnosis of schizophrenia. Lancet. 1995;346(8973):477-81.

2. Rado S. Dynamics and classification of disordered behavior. Am J Psychiatry. 1953;110(6):406-16.

3. Gottesman II, Gould TD. The endophenotype concept in psychiatry: etymology and strategic intentions. Am J Psychiatry. 2003;160(4):636-45.

4. Raine A. Schizotypal personality: neurodevelopmental and psychosocial trajectories. Annu Rev Clin Psychol. 2006;2:291-326.

5. Vollema MG, Postma B. Neurocognitive correlates of schizotypy in first degree relatives of schizophrenia patients. Schizophr Bull. 2002;28(3):367-77.

6. Dos Santos RG, Bouso JC, Hallak JEC. Serotonergic hallucinogens/ psychedelics could be promising treatments for depressive and anxiety disorders in end-stage cancer. BMC Psychiatry. 2019;19(1):321.

7. Baker KD, Skuse DH. Adolescents and young adults with $22 q 11$ deletion syndrome: psychopathology in an at-risk group. Bri J Psychiatry. 2005;186: $115-20$.

8. Stefanis NC, Van Os J, Avramopoulos D, Smyrnis N, Evdokimidis I, Hantoumi I, Stefanis CN. Variation in catechol-o-methyltransferase val158 met genotype associated with schizotypy but not cognition: a population study in 543 young men. Biol Psychiatry. 2004:56(7):510-5.

9. Ikeda M, Aleksic B, Kinoshita Y, Okochi T, Kawashima K, Kushima I, Ito Y, Nakamura Y, Kishi T, Okumura T, et al. Genome-wide association study of schizophrenia in a Japanese population. Biol Psychiatry. 2011;69(5):472-8.

10. Ripke S, Sanders AR, Kendler KS, Levinson DF, Sklar P, Holmans PA, Lin DY, Duan J, Ophoff RA, Andreassen OA, et al. Genome-wide association study identifies five new schizophrenia loci. Nat Genet. 2011;43(10):969-76.

11. Aberg KA, Liu Y, Bukszar J, McClay JL, Khachane AN, Andreassen OA, Blackwood D, Corvin A, Djurovic S, Gurling H, et al. A comprehensive familybased replication study of schizophrenia genes. JAMA Psychiatry. 2013;70(6): 573-81.

12. Luo XG, Klempan TA, Lappalainen J, Rosenheck RA, Charney DS, Erdos J, van Kammen DP, Kranzler HR, Kennedy JL, Gelernter J. NOTCH4 gene haplotype is associated with schizophrenia in African Americans. Biol Psychiatry. 2004; 55(2):112-7.

13. Wei J, Hemmings GP. The NOTCH4 locus is associated with susceptibility to schizophrenia. Nat Genet. 2000;25(4):376-7.

14. Ikeda M, Aleksic B, Yamada K, Iwayama-Shigeno Y, Matsuo K, Numata S, Watanabe Y, Ohnuma T, Kaneko T, Fukuo Y, et al. Genetic evidence for association between NOTCH4 and schizophrenia supported by a GWAS follow-up study in a Japanese population. Mol Psychiatry. 2013;18(6):636-8.

15. Tochigi M, Zhang X, Ohashi J, Hibino H, Otowa T, Rogers M, Kato T, Okazaki $Y$, Kato N, Tokunaga K. Association study between the TNXB locus and schizophrenia in a Japanese population. Am J Med Genet B Neuropsychiatr Genet. 2007;144(3):305-9.

16. Liu CM, Liu YL, Fann CSJ, Chenk WJ, Yang WC, Ouyang WC, Chen CY, Jou YS, Hsieh MH, Liu SK, et al. Association evidence of schizophrenia with distal genomic region of NOTCH4 in Taiwanese families. Genes Brain Behav. 2007; 6(6):497-502.

17. Zhang B, Fan QR, Li WH, Lu N, Fu DK, Kang YJ, Wang N, Li T, Wen XP, Li DX. Association of the NOTCH4 gene polymorphism rs204993 with schizophrenia in the Chinese Han population. Biomed Res Int. 2015;2015: 408096.

18. Chen J, Xu Y, Zhang J, Liu ZF, Xu C, Zhang KR, Shen Y, Xu Q. A combined study of genetic association and brain imaging on the DAOA gene in schizophrenia. Am J Med Genet B. 2013;162B(2):191-200.

19. Raichle ME, MacLeod AM, Snyder AZ, Powers WJ, Gusnard DA, Shulman GL. A default mode of brain function. Proc Natl Acad Sci U S A. 2001;98(2):67682.

20. Buckner RL, Sepulcre J, Talukdar T, Krienen FM, Liu HS, Hedden T, AndrewsHanna JR, Sperling RA, Johnson KA. Cortical hubs revealed by intrinsic functional connectivity: mapping, assessment of stability, and relation to Alzheimer's disease. J Neurosci. 2009;29(6):1860-73.

21. Zhu JJ, Zhuo CJ, Xu LX, Liu F, Qin W, Yu CS. Altered coupling between resting-state cerebral blood flow and functional connectivity in schizophrenia. Schizophr Bull. 2017;43(6):1363-74.

22. Wang L, Xia MR, Li K, Zeng YW, Su YN, Dai WJ, Zhang QG, Jin Z, Mitchell PB, Yu X, et al. The effects of antidepressant treatment on resting-state functional brain networks in patients with major depressive disorder. Hum Brain Mapp. 2015;36(2):768-78.

23. Chen X, Ji GJ, Zhu C, Bai X, Wang L, He K, Gao Y, Tao L, Yu F, Tian Y, et al. Neural correlates of auditory verbal hallucinations in schizophrenia and the therapeutic response to theta-burst Transcranial magnetic stimulation. Schizophr Bull. 2019;45(2):474-83.

24. Zhang Q, Shen J, Wu JL, Yu X, Lou WT, Fan HY, Shi L, Wang DF. Altered default mode network functional connectivity in schizotypal personality disorder. Schizophr Res. 2014;160(1-3):51-6.

25. Raine A. The SPQ: a scale for the assessment of schizotypal personality based on DSM-III-R criteria. Schizophr Bull. 1991;17(4):555-64.

26. Raine A, Reynolds C, Lencz T, Scerbo A, Triphon N, Kim D. Cognitiveperceptual, interpersonal, and disorganized features of schizotypal personality. Schizophr Bull. 1994;20(1):191-201.

27. Chen WJ, Hsiao CK, Lin CC. Schizotypy in community samples: the threefactor structure and correlation with sustained attention. J Abnorm Psychol. 1997;106(4):649-54.

28. Chao-Gan Y, Yu-Feng Z. DPARSF: a MATLAB toolbox for "pipeline" data analysis of resting-state fMRI. Front Syst Neurosci. 2010;4:13.

29. Song XW, Dong ZY, Long XY, Li SF, Zuo XN, Zhu CZ, He Y, Yan CG, Zang YF. REST: a toolkit for resting-state functional magnetic resonance imaging data processing. PLoS One. 2011;6(9):e25031.

30. Wei Q, Bai T, Chen Y, Ji G, Hu X, Xie W, Xiong Z, Zhu D, Wei L, Hu P, et al. The changes of functional connectivity strength in electroconvulsive therapy for depression: a longitudinal study. Front Neurosci. 2018;12:661.

31. Liang X, Zou Q, He Y, Yang Y. Coupling of functional connectivity and regional cerebral blood flow reveals a physiological basis for network hubs of the human brain. Proc Natl Acad Sci U S A. 2013;110(5):1929-34.

32. Justice NJ, Jan YN. Variations on the notch pathway in neural development. Curr Opin Neurobiol. 2002;12(1):64-70.

33. Fortini ME, Artavanis-Tsakonas S. Notch: neurogenesis is only part of the picture. Cell. 1993;75(7):1245-7.

34. Fiuza UM, Arias AM. Cell and molecular biology of notch. J Endocrinol. 2007; 194(3):459-74.

35. Sestan N, Artavanis-Tsakonas S, Rakic P. Contact-dependent inhibition of cortical neurite growth mediated by notch signaling. Science (New York). 1999;286(5440):741-6.

36. Benetti S, Novello L, Maffei C, Rabini G, Jovicich J, Collignon O. White matter connectivity between occipital and temporal regions involved in face and voice processing in hearing and early deaf individuals. Neurolmage. 2018; 179:263-74.

37. Cappadocia DC, Monaco S, Chen Y, Blohm G, Crawford JD. Temporal evolution of target representation, movement direction planning, and reach execution in occipital-parietal-frontal cortex: an fMRI study. Cereb Cortex. 2017;27(11):5242-60

38. Oestreich LKL, McCarthy-Jones S, Whitford TJ, Bank ASR. Decreased integrity of the fronto-temporal fibers of the left inferior occipito-frontal fasciculus associated with auditory verbal hallucinations in schizophrenia. Brain Imaging Behav. 2016;10(2):445-54.

39. van de Ven V, Jagiela AR, Oertel-Knochel V, Linden DEJ. Reduced intrinsic visual cortical connectivity is associated with impaired perceptual closure in schizophrenia. Neuroimage-Clin. 2017;15:45-52.

40. Zalesky A, Pantelis C, Cropley V, Fornito A, Cocchi L, McAdams H, Clasen L, Greenstein D, Rapoport JL, Gogtay N. Delayed development of brain connectivity in adolescents with schizophrenia and their unaffected siblings. JAMA Psychiatry. 2015;72(9):900-8.

41. Che XW, Wei DT, Li WF, Li HJ, Qiao L, Qiu J, Zhang QL, Liu YJ. The correlation between gray matter volume and perceived social support: a voxel-based morphometry study. Soc Neurosci-Uk. 2014;9(2):152-9.

42. Lingnau A, Downing PE. The lateral occipitotemporal cortex in action. Trends Cogn Sci. 2015;19(5):268-77.

43. Quadflieg S, Gentile F, Rossion B. The neural basis of perceiving person interactions. Cortex. 2015;70:5-20.

44. Giordano GM, Stanziano M, Papa M, Mucci A, Prinster A, Soricelli A, Galderisi S. Functional connectivity of the ventral tegmental area and avolition in subjects with schizophrenia: a resting state functional MRI study. Eur Neuropsychopharmacol. 2018;28(5):589-602.

45. Rigucci S, Rossi-Espagnet C, Ferracuti S, De Carolis A, Corigliano V, Carducci F, Mancinelli I, Cicone F, Tatarelli R, Bozzao A, et al. Anatomical substrates of cognitive and clinical dimensions in first episode schizophrenia. Acta Psychiatr Scand. 2013;128(4):261-70. 
46. Taylor SF, Chen AC, Tso IF, Liberzon I, Welsh RC. Social appraisal in chronic psychosis: role of medial frontal and occipital networks. J Psychiatr Res. 2011;45(4):526-38.

47. Liu B, Zhang XL, Hou B, Li J, Qiu CX, Qin W, Yu CS, Jiang TZ. The impact of MIR137 on dorsolateral prefrontal-hippocampal functional connectivity in healthy subjects. Neuropsychopharmacol. 2014;39(9):2153-60.

48. Xin-tian L. The distribution of left and right handedness in Chinese people. Acta Psychol Sin. 1983;03:268-76.

\section{Publisher's Note}

Springer Nature remains neutral with regard to jurisdictional claims in published maps and institutional affiliations.

Ready to submit your research? Choose BMC and benefit from:

- fast, convenient online submission

- thorough peer review by experienced researchers in your field

- rapid publication on acceptance

- support for research data, including large and complex data types

- gold Open Access which fosters wider collaboration and increased citations

- maximum visibility for your research: over $100 \mathrm{M}$ website views per year

At BMC, research is always in progress.

Learn more biomedcentral.com/submissions 